\title{
A Tractable Framework for Coverage and Outage in Heterogeneous Cellular Networks
}

\author{
Harpreet S. Dhillon, Radha Krishna Ganti and Jeffrey G. Andrews \\ Wireless Networking and Communications Group (WNCG), \\ Department of Electrical and Computer Engineering, \\ The University of Texas at Austin, \\ 1 University Station C0803, Austin, TX. \\ Email: \{dhillon@utexas.edu,rganti@austin.utexas.edu,jandrews@ece.utexas.edu\}
}

\begin{abstract}
We develop a tractable, flexible, and accurate model for downlink heterogeneous cellular networks. It consists of $K$ tiers of randomly-located base stations (BSs), where each tier may differ in terms of the average transmit power, the supported data rate and the BS density. This allows elements spanning traditional, micro, pico, and femtocell BSs to be simultaneously considered. Assuming a mobile user connects to its strongest BS, we derive its Signal-to-Interference-Ratio (SIR) distribution and use that to find the coverage (equivalently outage) probability over the entire network. We verify the accuracy of these analytical results through empirical comparisons with an actual $4 \mathrm{G}$ macrocell network.
\end{abstract}

\section{INTRODUCTION}

Cellular networks are in the process of a major transition, and will become increasingly complex over the next decade due to the co-deployment in space and frequency of very different classes of BSs. Traditional BSs will be joined by micro, pico, and femtocell BSs, as well as distributed antennas and out-of-band relays [1]-[6]. These heterogeneous cellular networks (HCNs) have BSs that differ by a few orders of magnitude in terms of their transmit power (and hence range) and the density in which they will be deployed. For example, it is easy to imagine a traditional cellular network having perhaps 10 pico-cells and 100 low-power femtocells in each highpower macro-cell, sharing the same licensed spectrum. Various plausible scenarios for $\mathrm{HCNs}$ are graphically illustrated in Figs. 1-4.

Clearly, the coverage, rate, and reliability that mobile users can achieve in such networks would seem to be quite different versus the familiar one-tier cellular networks of yesterday. There are two main challenges in understanding these multitier heterogeneous networks. First and foremost is to develop system models that capture the heterogeneity of these networks with enough accuracy to be realistic but enough simplicity to be useful. Second, is to be able to quantify important performance metrics like outage probability (versus an arbitrary Signal-to-Interference-plus-Noise-Ratio (SINR)), with the end goal of better understanding system design principles for HCNs. Solving these two problems is the goal of this paper.

Those familiar with cellular network analysis are likely to immediately recognize that these two goals are fairly ambitious, since even for traditional cellular networks, very sim- plified models must be used to gain tractability. For example, even to the present day the most popular analytical model is the incredibly simplistic Wyner model, which assumes channel gains from all the interfering BSs to be equal [8]. Although this model has a merit of being tractable, it is not accurate in most cases and does not even have a notion of outage since SIR is deterministic [9]. The most popular but largely intractable model is the two-dimensional hexagonal grid model, which is ubiquitous in textbooks and research alike. Typically, the grid model is used as the basis of system-level simulations and analysis is not possible [10]-[12]. However, the accuracy of such models in the case of random BS locations, such as those of femtocells, is questionable. A third way is to model the locations of BSs by a point process and then use techniques with foundations in stochastic geometry [13]-[16] to develop a tractable analytical model [17]. In [17], we showed perhaps surprisingly that not only does the added randomness result in a much more tractable framework, but even with the simple Poisson Point Process (PPP) it is about as accurate as the grid model.

The results in this paper can be viewed as an extension of [17] to a general downlink multi-tier network. This random model for the BS placements is likely even more sensible for a $K$-tier network since the BSs in lower tiers are more likely to mimic a random spatial distribution than the macro base stations. The model thus consists of $K$ tiers of PPP distributed BSs, where each tier may differ in terms of the average transmit power, the supported data rate, and the BS density (the average number of BSs per unit area). We assume each mobile connects to the strongest BS (open access), or the strongest one it is authorized to access (closed access).

The main result of the paper is a simple closed-form expression for the coverage probability, which is basically the probability that a randomly located mobile can achieve a target $\operatorname{SIR} \beta_{i}$ with at least one of the tiers. This coverage probability can be easily visualized as the complementary cumulative distribution function (CCDF) of the effective received SIR when all the tiers have the same SIR threshold $\beta$. We confirm the accuracy of our results and assumptions with simulations against an actual $4 \mathrm{G}$ macro-cell network (with lower tiers again modeled as Poisson distributed). 


\section{SySTEM MOdEL}

\section{A. Heterogeneous Cellular Network Model}

We model a $\mathrm{HCN}$ as a $K$-tier cellular network where each tier models the BSs of a particular type, such as those of femtocells or pico-cells. The BSs across tiers may differ in terms of the transmit power, the supported data rate and their spatial density. We assume that the BSs in the $i$-th tier are spatially distributed as a PPP $\Phi_{i}$ of density $\lambda_{i}$, transmit at a power $P_{i}$, and have a Signal-to-Interference-plus-Noise-Ratio (SINR) target of $\beta_{i}$. More precisely a mobile can reliably communicate with a BS $x$ in the $i$-th tier only if its downlink SINR with respect to that BS is greater than $\beta_{i}$. Thus, each tier can be uniquely defined by the tuple $\left\{P_{i}, \beta_{i}, \lambda_{i}\right\}$.

The mobiles are also modeled by an independent PPP $\Phi_{m}$ of density $\lambda_{m}$. Without loss of generality, we conduct analysis on a typical mobile user located at the origin. The fading (power) between a BS located at point $x$ and the typical mobile is denoted by $h_{x}$ and is assumed to be i.i.d exponential (Rayleigh fading). More complex channel distributions can be considered, at the price of decreased tractability, and we leave such generalizations to future work. The standard path loss function is given by $l(x)=\|x\|^{-\alpha}$, where $\alpha>2$ is the path loss exponent. Hence, the received power at a typical mobile user from a BS located at point $x_{i}$ (belonging to $i^{\text {th }}$ tier) is $P_{i} h_{x_{i}}\left\|x_{i}\right\|^{-\alpha}$, where $h_{x_{i}} \sim \exp (1)$. The resulting SIR expression assuming the user connects to this BS is:

$$
\operatorname{SIR}\left(x_{i}\right)=\frac{P_{i} h_{x_{i}}\left\|x_{i}\right\|^{-\alpha}}{\sum_{j=1}^{K} \sum_{x \in \Phi_{j} \backslash x_{i}} P_{j} h_{x}\|x\|^{-\alpha}} .
$$

Since self-interference dominates noise in all cellular networks of even modest density, we neglect thermal noise in this work, which further improves tractability. It was shown that adding noise has no affect on these results in the regimes of most interest in the 1-tier case [17], and we would expect that to hold even more strongly for a multi-tier network.

We consider the maximum SIR connectivity model, where each mobile user connects to its strongest BS, i.e., the BS that offers the highest received SIR. Mathematically the typical node at the origin is in coverage if:

$$
\max _{x \in \Phi_{i}} \operatorname{SIR}(x)>\beta_{i},
$$

for some $1 \leq i \leq K$. An important assumption we make is that the thresholds $\beta_{i}>1(0 \mathrm{~dB})$. The following Lemma shows that under this assumption, at most one BS in the entire network can provide SIR greater than the required threshold, which admits a simple form for the resulting coverage probability. Although some users in commercial cellular networks indeed have operating SIR below $0 \mathrm{~dB}$, they are in a distinct minority (cell edge users) and indeed later we show that this model holds very accurately at least to $-4 \mathrm{~dB}$, which covers even cell edge users.

Lemma 1. Given positive real numbers $\left\{a_{1}, a_{2} \ldots a_{n}\right\}$, which correspond to the received power from each $B S$ at the typical mobile user and defining $b_{i}=\frac{a_{i}}{\sum_{j \neq i} a_{j}}$, which corresponds to the SIR of the $i^{\text {th }} B S$, at most $m b_{i}$ 's can be greater than $1 / m$ for any positive integer $m$. As a special case of interest, only one $b_{i}$ can be greater than 1 and hence each randomly chosen mobile user has at most one BS with which it can successfully communicate.

Proof: See Appendix.

We will comment on the applicability of the derived results in the $\beta_{i}<0 \mathrm{~dB}$ regime in the Numerical Results section.

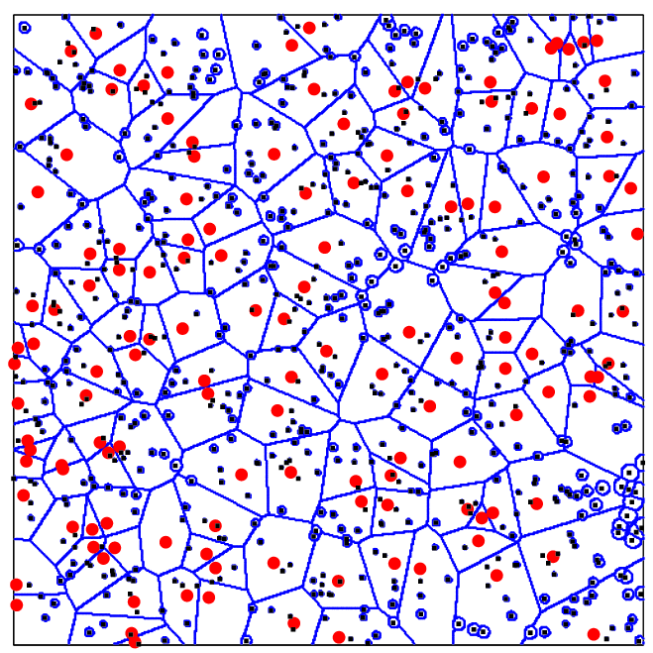

Fig. 1. Coverage regions in two-tier network. Both macro (red circles) and femto (black squares) BSs are distributed as independent PPPs. Also $P_{\text {femto }}=P_{\text {macro }} / 1000, \lambda_{\text {femto }}=5 \lambda_{\text {macro }}$.

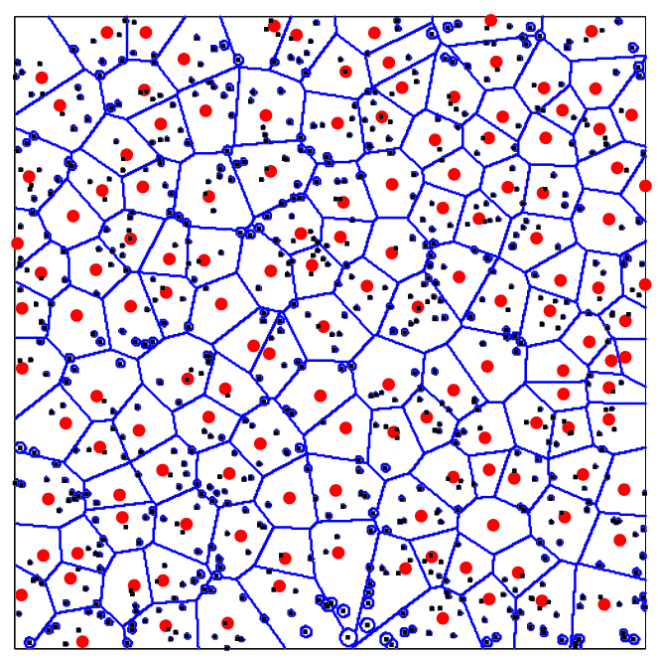

Fig. 2. Coverage regions in two-tier network. Macro BS locations (red circles) correspond to actual $4 \mathrm{G}$ deployment. Femto BSs (black squares) are distributed as PPP. Also $P_{\text {femto }}=P_{\text {macro }} / 1000, \lambda_{\text {femto }}=5 \lambda_{\text {macro }}$.

\section{B. Coverage Regions}

Before going into the analysis, let us first build a little intuition about the coverage regions in heterogeneous networks. Coverage regions are plotted in two steps. First, we randomly place $K$ different types of BSs on a 2-D plane according to the 
aforementioned independent PPPs. Second, the space is fully tessellated following the maximum SIR connectivity model. Due to the differences in the transmit powers over the tiers, the coverage plots do not correspond to a classical Voronoi tessellation (also called Dirichlet tessellation) [18]. In fact, they closely resemble a circular Dirichlet tessellation, which is also called a multiplicatively weighted Voronoi diagram, where the distance between the points (BSs) is multiplied by positive "weights" [19]. The coverage regions for a two-tier network - for example comprising macro and femtocells are depicted in Figs. 1 and 2 for two cases: 1) the macro-cell BSs are distributed according to PPP (our model), and 2) the macro-cell BSs correspond to an actual $4 \mathrm{G}$ deployment over a relatively flat urban region. The femtocells are distributed according to an independent PPP in both cases. Qualitatively, the coverage regions are quite similar in the two cases. The random model used in this paper could likely be further improved by incorporating a point process that models repulsion or minimum separation distance, such as determinantal and Matern processes [14], [15], but we leave such extensions (which will erode tractability) to future work. We will provide more quantitative support of our model in the numerical results section.

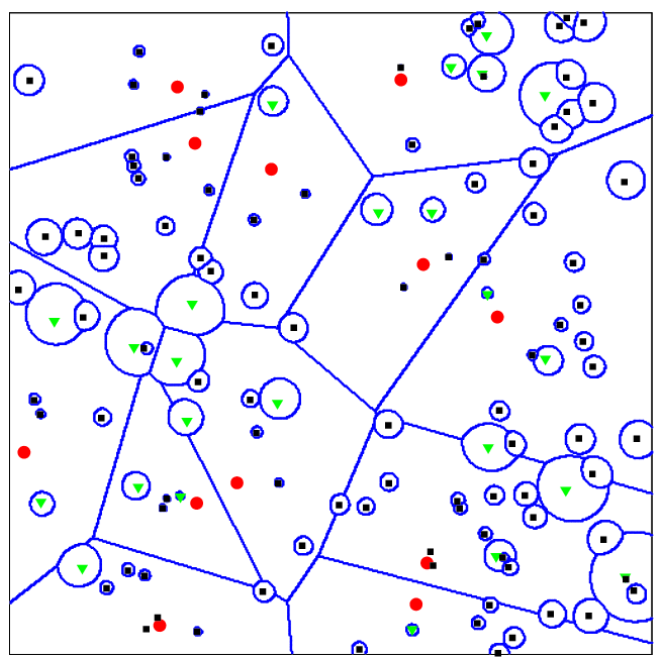

Fig. 3. Coverage regions in three-tier network. All the tiers, i.e., macro (red circles), pico (green triangles), femto (black squares), are modeled as independent PPPs. $P_{\text {pico }}=P_{\text {macro }} / 100, P_{\text {femto }}=P_{\text {macro }} / 1000$, $\lambda_{\text {pico }}=2 \lambda_{\text {macro }}, \lambda_{\text {femto }}=8 \lambda_{\text {macro }}$.

In Figs. 3 and 4 , the coverage regions are now shown with an additional pico-cell tier. As is the case in the actual networks, we assume that the macro-cells have the highest and the femtocells have the smallest transmit powers, with pico-cells in between. Therefore, the coverage regions for the femtocells are in general much smaller than the other two tiers, particularly when they are nearby a higher power BS. Similarly, we observe that the coverage footprint of picocells increases when they are farther from the macro BSs. These observations highlight the particularly important role of smaller cells on the cell edges.

\section{SIR Distribution AND COVERAge Probability}

A typical mobile user is said to be under coverage if it is able to connect to at least one of the BSs. In the case when all the tiers have same SIR threshold $\beta>1$, coverage probability can be easily visualized as the complementary cumulative distribution function (CCDF) of the effective received SIR. With this understanding, we now derive the probability of coverage for a randomly located mobile user both for the open and closed access strategies.

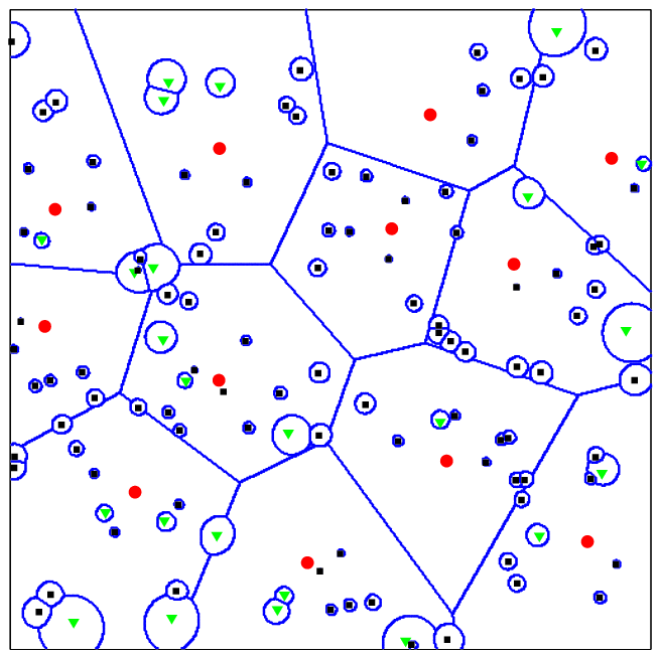

Fig. 4. Coverage regions in three-tier network. Macro BS locations (red circles) correspond to actual $4 \mathrm{G}$ deployment. Pico (green triangles) and femto (black squares) BSs are distributed as independent PPPs. Also $P_{\text {pico }}=P_{\text {macro }} / 100, P_{\text {femto }}=P_{\text {macro }} / 1000, \lambda_{\text {pico }}=2 \lambda_{\text {macro }}$, $\lambda_{\text {femto }}=8 \lambda_{\text {macro }}$.

\section{A. Open Access}

We first assume the open access strategy where a typical mobile user is allowed to connect to any tier without any restriction. The main result for the probability of coverage in this setup is given by Theorem 1 .

Theorem 1. The coverage probability for a typical randomly located mobile user assuming Rayleigh fading for all the links is:

$\mathrm{P}_{\mathrm{c}}\left(\left\{\lambda_{i}\right\},\left\{\beta_{i}\right\},\left\{P_{i}\right\}\right)=\frac{\pi}{C(\alpha)} \frac{\sum_{i=1}^{K} \lambda_{i} P_{i}^{2 / \alpha} \beta_{i}^{-2 / \alpha}}{\sum_{i=1}^{K} \lambda_{i} P_{i}^{2 / \alpha}}, \quad \beta_{i}>1$,

where $C(\alpha)$ can be expressed as:

$$
C(\alpha)=\frac{2 \pi^{2} \csc \left(\frac{2 \pi}{\alpha}\right)}{\alpha} .
$$

Proof: The coverage probability in a $K$-tier network under maximum SIR connectivity model is

$$
\mathrm{P}_{\mathrm{c}}=\mathbb{P}\left(\bigcup_{i \in K, x_{i} \in \Phi_{i}} \operatorname{SIR}\left(x_{i}\right)>\beta_{i}\right)
$$




$$
\begin{aligned}
& =\mathbb{E}\left[\mathbf{1}\left(\bigcup_{i \in K, x_{i} \in \Phi_{i}} \operatorname{SIR}\left(x_{i}\right)>\beta_{i}\right)\right] \\
& \stackrel{(a)}{=} \sum_{i=1}^{K} \mathbb{E} \sum_{x_{i} \in \Phi_{i}}\left[\mathbf{1}\left(\operatorname{SIR}\left(x_{i}\right)>\beta_{i}\right)\right] \\
& \stackrel{(b)}{=} \sum_{i=1}^{K} \lambda_{i} \int_{\mathbb{R}^{2}} \mathbb{P}\left(\frac{P_{i} h_{x_{i}} \ell\left(x_{i}\right)}{I_{x_{i}}}>\beta_{i}\right) \mathrm{d} x_{i} \\
& \stackrel{(c)}{=} \sum_{i=1}^{K} \lambda_{i} \int_{\mathbb{R}^{2}} \mathbb{E}\left[\exp \left(-\frac{\beta_{i} I_{x_{i}}}{P_{i} \ell\left(x_{i}\right)}\right)\right] \mathrm{d} x_{i} \\
& =\sum_{i=1}^{K} \lambda_{i} \int_{\mathbb{R}^{2}} \mathcal{L}_{I_{x_{i}}}\left(\frac{\beta_{i}}{P_{i} l\left(x_{i}\right)}\right) \mathrm{d} x_{i},
\end{aligned}
$$

where $(a)$ follows from Lemma 1 under the assumption that $\beta_{i}>1 \forall i,(b)$ follows from Campbell Mecke Theorem and $(c)$ follows from the fact that the channel gains are assumed to be Rayleigh distributed. Here $\mathcal{L}_{I_{x_{i}}}($.$) is the Laplace transform$ of the cumulative interference from all the tiers when the randomly chosen mobile user is being served by the $i^{\text {th }}$ tier. Since the point processes are stationary, the net interference does not depend on the location $x_{i}$. Therefore, we denote $\mathcal{L}_{I_{x_{i}}}$ by $\mathcal{L}_{I_{i}}$ which is given by

$$
\begin{aligned}
\mathcal{L}_{I_{i}}(s) & =\mathbb{E}\left[\exp \left(-s I_{x_{i}}\right)\right] \\
& =\prod_{j=1}^{K} \mathbb{E}\left[\prod_{x_{j} \in \Phi_{j} / x_{i}} \exp \left(-s P_{j} h_{x_{j}} l\left(x_{j}\right)\right)\right] \\
& =\prod_{j=1}^{K} \mathbb{E}_{\Phi_{j}}\left[\prod_{x_{j} \in \Phi_{j} / x_{i}} \mathbb{E}_{h}\left[\exp \left(-s P_{j} h_{x_{j}} l\left(x_{j}\right)\right)\right]\right] \\
& \stackrel{(a)}{=} \prod_{j=1}^{K} \mathbb{E}_{\Phi_{j}}\left[\prod_{x_{j} \in \Phi_{j} / x_{i}} \frac{1}{1+s P_{j} l\left(x_{j}\right)}\right] \\
& \stackrel{(b)}{=} \prod_{j=1}^{K} \exp \left(-2 \pi \lambda_{i} \int_{0}^{\infty}\left(1-\frac{1}{1+s P_{j} r^{-\alpha}}\right) r \mathrm{~d} r\right) \\
& \stackrel{(c)}{=} \prod_{j=1}^{K} e^{\left(-2 \pi \lambda_{i}\left(s P_{j}\right)^{2 / \alpha} \int_{0}^{\infty} r \int_{0}^{\infty} \exp \left(-t\left(1+r^{\alpha}\right)\right) d t \mathrm{~d} r\right)}
\end{aligned}
$$

where $(a)$ follows from the Rayleigh fading assumption (i.e., $h \sim \exp (1)),(b)$ follows by PGFL and, (c) results from simple algebraic manipulation. Using the properties of Gamma function, (5) can be further simplified to

$$
\mathcal{L}_{I}(s)=\exp \left(-s^{2 / \alpha} C(\alpha) \sum_{i=1}^{k} \lambda_{i} P_{i}^{2 / \alpha}\right)
$$

where $C(\alpha)$ is the Baccelli's constant and

$$
C(\alpha)=\frac{2 \pi^{2} \csc \left(\frac{2 \pi}{\alpha}\right)}{\alpha}
$$

Using (6) and (4) the coverage probability $P_{c}$ is

$$
\begin{aligned}
\mathrm{P}_{\mathrm{c}}\left(\left\{\lambda_{i}\right\},\left\{\beta_{i}\right\},\left\{P_{i}\right\}\right) & =\sum_{i=1}^{K} \lambda_{i} \int_{\mathbb{R}^{2}} \mathcal{L}_{I_{x_{i}}}\left(\frac{\beta_{i}}{P_{i} l\left(x_{i}\right)}\right) \mathrm{d} x_{i} \\
& =\frac{\pi}{C(\alpha)} \frac{\sum_{i=1}^{K} \lambda_{i} P_{i}^{2 / \alpha} \beta_{i}^{-2 / \alpha}}{\sum_{i=1}^{K} \lambda_{i} P_{i}^{2 / \alpha}} .
\end{aligned}
$$

This completes the proof.

Setting $K=1$, leads back to the single-tier case and leads to the following corollary.

Corollary 1. In a single tier cellular network (i.e., $K=1$ ), $\mathrm{P}_{\mathrm{c}}(\lambda, \beta, P)$ can be expressed as:

$$
\mathrm{P}_{\mathrm{c}}(\lambda, \beta, P)=\frac{\pi}{C(\alpha) \beta^{2 / \alpha}}
$$

This corollary states that $\mathrm{P}_{c}$ in a single-tier network is independent of the density of the BSs and is solely dependent upon the SIR threshold value. This is consistent with the fact that increasing the density of the BSs decreases the distance of the typical mobile from the BS to which it is connected and hence increases the received power. At the same time, it decreases the distance of the mobile from the interferes with the same factor and hence increases the interference power with the same factor. Perhaps surprisingly, both the effects cancel each other and hence coverage probability does not depend on the density of BSs.

Corollary 2. Assuming $\beta_{i}=\beta \forall i, \mathrm{P}_{\mathrm{c}}\left(\left\{\lambda_{i}\right\}, \beta,\left\{P_{i}\right\}\right)$ can be expressed as:

$$
\mathrm{P}_{\mathrm{c}}\left(\left\{\lambda_{i}\right\}, \beta,\left\{P_{i}\right\}\right)=\frac{\pi}{C(\alpha) \beta^{2 / \alpha}}
$$

This is perhaps an unexpected result since it states that the coverage probability is not affected by the number of tiers. In fact, it is exactly the same as that of the single-tier case. Therefore, more BSs can be added in any tier without affecting the coverage and hence the net network capacity can be increased linearly with the number of BSs.

\section{B. Closed Access}

Under closed access scheme, a mobile user is allowed to connect to only a subset of tiers and the rest of the tiers act purely as interferers. The main result of coverage probability under closed access is given by Proposition 1.

Proposition 1. Assuming a mobile user is allowed to connect to only a subset $\mathcal{B}$ of the $K$ tiers, the coverage probability can be expressed as:

$$
\mathrm{P}_{\mathrm{c}}\left(\left\{\lambda_{i}\right\},\left\{\beta_{i}\right\},\left\{P_{i}\right\}\right)=\frac{\pi}{C(\alpha)} \frac{\sum_{i \in \mathcal{B}} \lambda_{i} P_{i}^{2 / \alpha} / \beta_{i}^{2 / \alpha}}{\sum_{i=1}^{K} \lambda_{i} P_{i}^{2 / \alpha}},
$$

Proof: The proof follows directly from the proof of Theorem 1.

Corollary 3. Assuming the threshold of each tier to be same (and equal to $\beta$ ) and the transmit power of each tier to be same 
(and equal to $P$ ), the coverage probability can be expressed as:

$$
\mathrm{P}_{\mathrm{c}}\left(\left\{\lambda_{i}\right\}, \beta,\left\{P_{i}\right\}\right)=\frac{\pi}{C(\alpha) \beta^{2 / \alpha}} \frac{\sum_{i \in \mathcal{B}} \lambda_{i}}{\sum_{i=1}^{K} \lambda_{i}}
$$

Above corollary states that if the thresholds and transmit powers of all the tiers are same, closed access has a lower coverage than open access by a factor of $\frac{\sum_{i \in \mathcal{B}} \lambda_{i}}{\sum_{i=1}^{K} \lambda_{i}}$.

\section{Numerical Results}

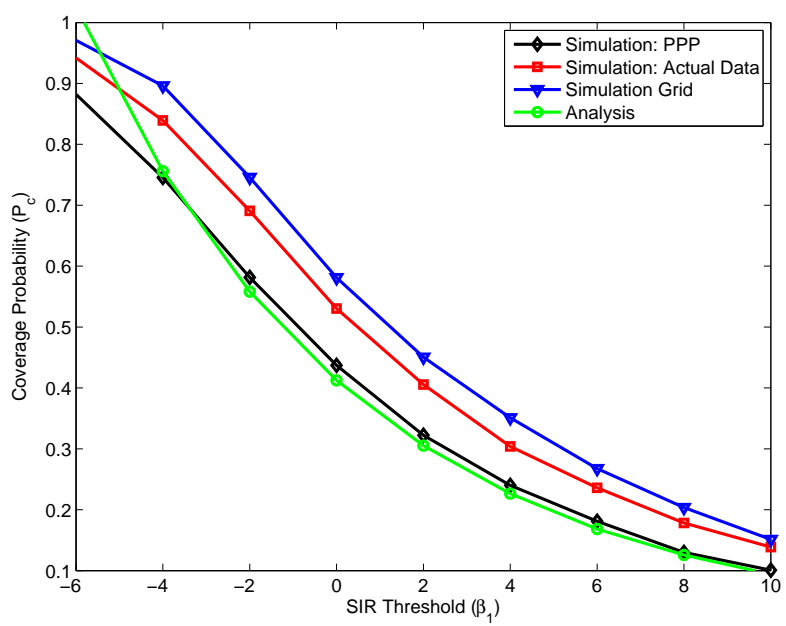

Fig. 5. Coverage probability in a two-tier $\operatorname{HCN}\left(K=2, \alpha=3, P_{1}=1000\right.$, $P_{2}=1, \lambda_{2}=2 \lambda_{1}, \beta_{2}=1 \mathrm{~dB}$ ).

While a random PPP model is the best that can be hoped for in modeling "unplanned" tiers, such as femtocells, its accuracy in modeling "planned" BS locations, such as those of macrocells, is open to question. Therefore, we begin this section by verifying the PPP assumption for macro-cells from a coverage probability perspective. To achieve this, we consider a two-tier network in three different scenarios: 1) the macro-cell BSs are distributed according to PPP (our model), 2) the macro-cell BSs correspond to an actual $4 \mathrm{G}$ deployment, and 3) macrocell BSs are distributed according to hexagonal grid model. The second tier is modeled as an independent PPP in all the three cases. We compare the coverage probabilities for these scenarios in Fig. 5. We observe that the actual coverage probability lies in between the coverage probabilities achieved by the PPP and the grid model. This is due to the fact that the likelihood of having a dominant interferer is highest in PPP and least in grid model, with the real scenario being inbetween. This comparison shows that the PPP assumption is nearly as accurate as the grid model in the case of macrocells, with PPP providing a lower bound and grid model providing an upper bound to the actual coverage probability. We now validate the main coverage probability result, given by Theorem 1, by comparing it with the simulation result in Fig. 5. It should be noted that $\mathrm{P}_{c}$ given by Theorem 1 is an exact solution to coverage probability when $\beta_{i}>1 \forall i$ but is an upper bound on the coverage probability when $\beta_{i}<1$. As expected, theoretical and simulated results match reasonably well for $\beta_{i}>1$ but interestingly, the theoretical results also provide a tight upper bound to the exact solution even until $\beta_{1}=-4 \mathrm{~dB}(\approx 4)$. This suggests that under the given simulation parameters, the likelihood of connecting to multiple BSs even through $\beta_{1}=-4 \mathrm{~dB}$ is very low.

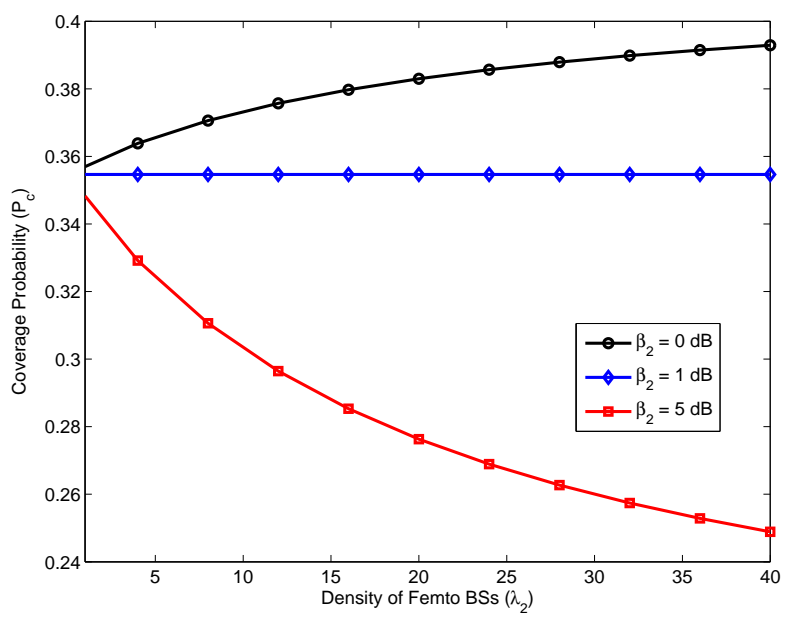

Fig. 6. Effect of changing the density of femto-cell BSs on the coverage probability in a two-tier $\mathrm{HCN}\left(K=2, \alpha=3, P_{1}=10, P_{2}=.1, \lambda_{1}=1\right.$, $\left.\beta_{1}=1 \mathrm{~dB}\right)$.

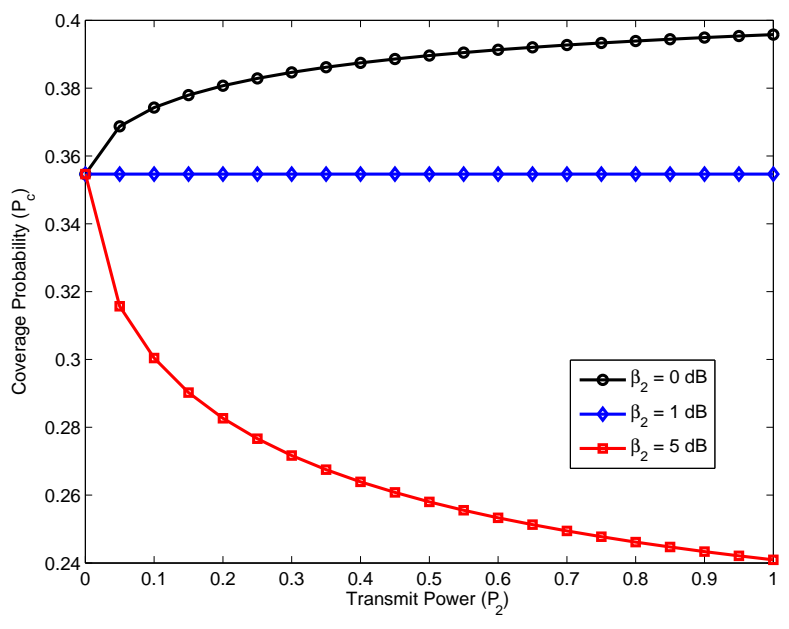

Fig. 7. Effect of changing the transmit power of femto-cell BSs on the coverage probability in a two-tier $\operatorname{HCN}\left(K=2, \alpha=3, P_{1}=100, \lambda_{1}=1\right.$, $\left.\lambda_{2}=50, \beta_{1}=1 \mathrm{~dB}\right)$.

After validating the main result, we again look at the coverage probability in the context of a two-tier HCN where a femtocell network is overlaid on a conventional macro-cell network. In particular, we study the effect of BS density and transmit power of the femtocell tier on the overall coverage probability in Figs. 6 and 7, respectively. We note that the coverage probability has a strong dependence on the SIR threshold of the femtocell tier. In fact, coverage probability can be theoretically improved if the femtocell tier threshold $\beta_{2}$ is lower than that of the macro-cell tier threshold $\beta_{1}$. 
This is due to the fact that more BSs can now connect to the femtocell tier due to a lower threshold, hence leading to an improved coverage. On the other hand, the addition of femtocell tier reduces the coverage probability if the SIR threshold of femtocell tier is higher than that of the macro-cell tier.

\section{CONCLUSION}

In this paper, we have developed a tractable model for $K$ tier downlink HCNs. The BS locations of each tier are modeled by an independent PPP. The BSs across tiers may differ in terms of the transmit power, the supported data rate and the BS density. While a random model is the best that can be hoped for in modeling "unplanned" tiers, such as femtocells, we have shown that this model is also as good as the popular grid model for modeling the "planned" tiers, such as macro-cells, by comparing it with an actual $4 \mathrm{G}$ macro-cell deployment. Using this model, we have obtained simple closed form expressions for the coverage probability of a randomly located mobile user in both the open and the closed access scenarios.

Future extensions to our approach from physical layer perspective could be in the study of HCNs that employ multiple antennas, spread spectrum, power control, interference cancelation or interference alignment. From the MAC layer perspective, it would be interesting to include scheduling, resource allocation and frequency reuse in our setup.

\section{REFERENCES}

[1] Chih-Lin I, L.J. Greenstein and R.D. Gitlin, "A Microcell/Macrocell Cellular Architecture for Low- and High-Mobility Wireless Users", IEEE J. Sel. Areas in Communications, vol. 11, no. 6, pp. 885-891, Aug. 1993.

[2] V. Chandrasekhar, J. G. Andrews, and A. Gatherer, "Femtocell Networks: A Survey", IEEE Comm. Magazine, vol. 46, no. 9, pp. 59-67, Sept. 2008.

[3] Picochip, "The Case For Home Base Stations", White Paper, April 2007.

[4] V. Chandrasekhar and J. G. Andrews, "Uplink Capacity and Interference Avoidance for Two-Tier Femtocell Networks", IEEE Trans. Wireless Comm., vol. 8, no. 7, pp. 3498-3509, July 2009.

[5] J. Zhang and J. G. Andrews, "Distributed Antenna Systems with Randomness", IEEE Trans. Wireless Comm., vol. 7, no. 9, pp. 36363646, Sept. 2008.

[6] S. Kishore, "Capacity and Coverage in two-tier Cellular CDMA Networks", Ph.D. Dissertation, Princeton University, 2003.

[7] Qualcomm R\&D, "LTE Advanced: Heterogeneous Networks", white paper, Qualcomm Inc., 2008.

[8] A. D. Wyner, "Shannon-Theoretic Approach to a Gaussian Cellular Multiple-Access Channel", IEEE Trans. Inform. Th., vol. 40, no. 11 , pp. 1713-1727, Nov. 1994.

[9] J. Xu, J. Zhang, and J. G. Andrews, "On the Accuracy of the Wyner Model in Cellular Networks", submitted. [Online] Available: http://arxiv.org/abs/1009.5900.

[10] S. Catreux, L. J. Greenstein, and P. F. Dressen, "Simulation Results for an Interference-Limited Multiple-Input Multiple-Output Cellular System", IEEE Comm. Lett., vol. 4, pp. 334-336, Nov. 2000.

[11] A. Ganz, C. M. Krishna, D. Tang, and Z. J. Haas, "On Optimal Design of Multitier Wireless Cellular Systems", IEEE Comm. Magazine, vol. 35, no. 2, pp. 88-93, Feb. 1997.

[12] E. Ekici and C. Ersoy, "Multi-Tier Cellular Network Dimensioning", ACM Wireless Networks, vol. 7, no. 4, pp. 401-411, July 2001.

[13] F. Baccelli, M. Klein, M. Lebourges and S. Zuyev, "Stochastic Geometry and Architecture of Communication Networks", Telecommunication Systems, vol. 7, no. 1, pp. 209-227, 1997.

[14] D. Stoyan, W. S. Kendall, and J. Mecke, Stochastic Geometry and its Applications. Wiley series in probability and mathematical statistics. Wiley, New York, $2^{\text {nd }} e d ., 1995$.
[15] F. Baccelli and B. Blaszczyszyn, Stochastic Geometry and Wireless Networks. NOW: Foundations and Trends in Networking, 2010.

[16] M. Haenggi, J. G. Andrews, F. Baccelli, O. Dousse, and M Franceschetti, "Stochastic Geometry and Random Graphs for the Analysis and Design of Wireless Networks", IEEE J. Sel. Areas in Communications, vol. 27, no. 7, pp. 1029-46, Sept. 2009.

[17] J. G. Andrews, F. Baccelli and R. K. Ganti, "A Tractable Approach to Coverage and Rate in Cellular Networks", submitted to IEEE Trans. Comm. [Online] Available: http://arxiv.org/abs/1009.0516.

[18] F. Aurenhammer, "Voronoi Diagrams - A Survey of a Fundamental Geometric Data Structure", ACM Computing Surveys, vol. 23, no. 3, pp. 345-405, Sept. 1991.

[19] P. F. Ash and E. D. Bolker, "Generalized Dirichlet Tessellations", Geometriae Dedicata, vol. 20, no. 2, pp. 209-243, 1986.

\section{APPENDIX}

PROOF OF LEMMA 1

$$
\begin{aligned}
b_{i} & =\frac{a_{i}}{\sum_{j \neq i} a_{j}}=\frac{a_{i}}{\sum_{j} a_{j}-a_{i}} \\
& \Rightarrow \frac{b_{i}}{1+b_{i}}=\frac{a_{i}}{\sum_{j} a_{j}} \\
& \Rightarrow \sum_{i=1}^{n} \frac{b_{i}}{1+b_{i}}=1 \\
& \Rightarrow \sum_{i=1}^{n} \frac{1}{1 / b_{i}+1}=1 .
\end{aligned}
$$

We first prove the result for $m=1$ (by contradiction) and then show that it can be trivially extended to the case of a general $m$.

We first observe that (13) is satisfied if only one of the $b_{i}$ 's is greater than 1 . Now assume that two $b_{i}$ 's are greater than one and without loss of generality, assume that they are $b_{1}$ and $b_{2}$. This implies $1 / b_{1}$ and $1 / b_{2} \in(0,1)$. Therefore, $\frac{1}{1 / b_{i}+1}$ and $\frac{1}{1 / b_{i}+1} \in(1 / 2,1)$. Thus,

$$
\begin{aligned}
\sum_{i=1}^{n} \frac{1}{1 / b_{i}+1} & =\sum_{i=1}^{2} \frac{1}{1 / b_{i}+1}+\sum_{i=3}^{n} \frac{1}{1 / b_{i}+1}, \\
& >1+\sum_{i=3}^{n} \frac{1}{1 / b_{i}+1},
\end{aligned}
$$

which is in contradiction with (13). Since (13) does not even hold for two $b_{i}$ 's greater than one, it proves that the only one of the $b_{i}$ 's can be greater than one. Similarly for the case of general $m$, it is easy to observe that (13) is trivially satisfied if at most $m$ of the $b_{i}$ 's are greater than $1 / m$. Now assume that $m+1 b_{i}$ 's are greater than $1 / m$ and without loss of generality, assume that they are $b_{1}, b_{2}, \ldots, b_{m+1}$. Proceeding as in (14),

$$
\sum_{i=1}^{n} \frac{1}{1 / b_{i}+1}>1+\sum_{i=m+2}^{n} \frac{1}{1 / b_{i}+1},
$$

which is in contradiction to (13). Therefore, at most $m b_{i}$ 's can be greater than $1 / m$. 